\title{
Almost perfect powers in consecutive integers
}

\author{
by \\ G. Hanrot (Nancy), N. SARAdha (Mumbai) \\ and T. N. Shorey (Mumbai)
}

1. Introduction. For an integer $\nu>1$, we write $P(\nu)$ for the greatest prime factor of $\nu$ and we put $P(1)=1$. Let $b, k \geq 2, \ell>2, n$ and $y$ be positive integers such that $P(b) \leq k$ and $\ell$ is prime. Let $t \geq 2$ and $r \in\{0,1\}$ be given by $t=k-r$. Thus $k \geq 2$ if $r=0$ and $k \geq 3$ if $r=1$. Further, let $d_{1}<\ldots<d_{t}$ be integers in the interval $[0, k)$. We consider the equation

$$
\left(n+d_{1}\right) \ldots\left(n+d_{t}\right)=b y^{\ell}
$$

in integers $b, k, \ell, n, y, d_{1}, \ldots, d_{t}$ as above. If $r=0$, then $d_{i}=i$ for $0 \leq i<k$ and the left hand side of (1) is $n(n+1) \ldots(n+k-1)$. If $r=1$, the left hand side of (1) is obtained by omitting a term $n+i$ for some $i$ with $0 \leq i<k$ from $\{n, n+1, \ldots, n+k-1\}$. For considering equation (1), it is natural to suppose that the left hand side of (1) is divisible by a prime exceeding $k$. This implies that $n>k^{\ell}$, which we assume throughout the paper without reference. For an account of results on equation (1) and its extensions, we refer to [13] and [14].

Erdős and Selfridge [4] proved that equation (1) with $r=0$ and $P(b)<k$ does not hold. The assumption $P(b)<k$ has been relaxed to $P(b) \leq k$ for $k \geq 4$ by Saradha [11] and for $k=2,3$ by Györy [5]. The proof of Saradha depends on the method of Erdős and Selfridge whereas Győry derived his results from the theorems of Ribet and Darmon and Merel on generalised Fermat equation. Saradha [11] showed that equation (1) with $r=1$ implies that $k \leq 8$. The results of Györy and Saradha are stated with the assumption that the left hand side of (1) is divisible by a prime exceeding $k$. But it is clear from their proofs that this assumption can be relaxed to $n>k^{\ell}$. Saradha and Shorey [12] proved that equation (1) with $r=1$ and $b=1$ never holds. The assumption $n>k^{\ell}$ is not required in the preceding result if $\left(n, k, d_{1}, \ldots, d_{t}\right)=(2,3,0,2),(1,4,0,1,3)$ are excluded. In this paper, we relax the assumption $k \leq 8$ in the result of Saradha stated above.

2000 Mathematics Subject Classification: Primary 11D61. 
Theorem. Let $n>k^{\ell}$. Then equation (1) with $r=1$ and $k \in\{6,7,8\}$ does not hold. This is also the case for $k=3,5$ if $P(b)<k$.

The cases $k=3,4,5$ if $P(b) \leq k$ and $k=4$ if $P(b)<k$ remain open. It is possible to settle the cases $k=3,4,5$ with $P(b) \leq k$ if we can solve completely a generalised Fermat equation of the form $A x^{\ell}+B y^{\ell}+C z^{\ell}=0$ with $P(A B C) \leq 3$ in integers $\ell \geq 3, x, y$ and $z$. It is clear from the proof of the Theorem that the case $k=4, P(b)<k$ can be settled if we solve an equation of the form $2^{\alpha} x^{\ell}+3^{\beta} y^{\ell}+z^{\ell}=0$ with $0 \leq \alpha \leq 3$ in integers $\ell \geq 3, x, y, z$ such that $\operatorname{gcd}(6, x y z)=\operatorname{gcd}(x, y, z)=1$. The Theorem is obtained by combining the elementary method of Erdös and Selfridge with the contributions of Wiles, Ribet, Darmon and Merel and others on the generalised Fermat equation and the developments of Bilu and Hanrot on solving Thue equations by Baker's method. For a prime $p>k$, we write $b_{p}$ for $b$ times a power of $p$. Then we conclude that equation (1) with $r=0$, $n>k^{\ell}, b$ replaced by $b_{p}$ and $k \geq 6$ is not possible. For this, we apply the result of Saradha and the Theorem to the equation obtained from (1) with $r=0$ by deleting a factor $n+i$ with $0 \leq i<k$ on the left hand side which is divisible by $p$. In particular, we derive the following result where $p^{(k)}$ denotes the least prime exceeding $k$.

Corollary. Let $n>k^{\ell}$. Then equation (1) with $r=0, P(b) \leq p^{(k)}$ and $k \geq 6$ does not hold.

For the proof of our Theorem, it is easily seen that we can restrict ourselves to the case of an equation $m(m+i)(m+j)=b y^{\ell}$ for a few small values of $i$ and $j$. A careful study of the possible values of $m, m+i$ and $m+j$ then reduces in turn the preceding equation to a small number of Thue equations for each $\ell$ (Lemmas 6-12), which are solved by Baker's method (Lemmas 13-14), as shown e.g. in [2]. To apply Baker's method, we need to keep a check on the degree as well as on the coefficients of the Thue equations. The degree is found to be $\leq 17$ by the elementary method of Erdös and Selfridge (see Lemma 1) and a check on the coefficients is provided by contributions on the generalised Fermat equation (Lemmas 2-5). We shall prove analogous results for equation (1) and more general equations with $\ell=2$ in a subsequent paper.

2. Lemmas. In this section, we give the lemmas for the proof of the Theorem. In these lemmas, the letter $b$ is used in a context which is different from that of Section 1 but this will be clear and it should not cause any confusion. Further, we shall understand, without reference, that $\ell$ is a prime number $>2$ in these lemmas. Let $2=p_{1}<p_{2}<\ldots$ denote the sequence of all primes. For any integer $m \geq 1$, we define as in [11, p. 159], 


$$
f_{0}(k, m)=t-\sum_{h \geq m+1}\left(\left[\frac{k}{p_{h}}\right]+\varepsilon_{h}\right)
$$

where $\varepsilon_{h}=0$ if $p_{h}>k$ and for $p_{h} \leq k, \varepsilon_{h}=0$ or 1 according as $p_{h} \mid k$ or not for $h \geq m+1$. We begin with an elementary result due to Erdős and Selfridge [4] which is fundamental in their method.

Lemma 1 . Let $1 \leq \ell^{\prime} \leq \ell-1$ and $f_{0}(k, m)$ be as above. Then equation (1) implies that

$$
\left(\begin{array}{c}
f_{0}(k, m)+\ell^{\prime}-1 \\
\ell^{\prime}
\end{array}\right) \leq \ell^{m}
$$

where the left hand side is zero if $f_{0}(k, m)<1$.

The next result on a generalised Fermat equation is due to Ribet [9] for $\alpha>1$ and Darmon and Merel [3] for $\alpha=1$.

Lemma 2. Let $\alpha$ be an integer with $1 \leq \alpha<\ell$. Then the equation

$$
x^{\ell}+y^{\ell}=2^{\alpha} z^{\ell}
$$

in non-zero relatively prime integers $x, y, z$ has no solution for $\alpha>1$ and for $\alpha=1$, it has only the trivial solution for which $x y z= \pm 1$.

By using the contributions of Wiles, Ribet and others the following result on a more generalised Fermat equation has been given in Sander [10] and in [12, Lemma 13].

Lemma 3. Let $\ell \geq 5$. Let $a, b, c$ be non-zero integers such that either $P(a b c) \leq 3$ or $a, b, c$ are composed of only 2 's and 5 's. Then the equation

$$
\begin{array}{ll}
a x^{\ell}-b y^{\ell}=c z^{\ell} \quad \text { in non-zero integers } x, y, z \text { with } \\
& \operatorname{gcd}\left(a x^{\ell}, b y^{\ell}, c z^{\ell}\right)=1, \operatorname{ord}_{2}\left(b y^{\ell}\right) \geq 4
\end{array}
$$

has no solution.

The next result of Bennett [1] is based on the hypergeometric method.

Lemma 4. For non-zero integers $a$ and $b$, the equation

$$
\left|a x^{\ell}-b y^{\ell}\right|=1
$$

has at most one solution in positive integers $(x, y)$. Hence the equation

$$
\left|(a+1) x^{\ell}-a y^{\ell}\right|=1
$$

has the only solution $(x, y)=(1,1)$ in positive integers.

Györy [5] derived from Lemma 2 the following result.

Lemma 5. Let $P(b) \leq 3$. The solutions of equation

$$
m(m+1)(m+2)=b y^{\ell} \quad \text { in positive integers } m \text { and } y
$$

are given by $m=1,2$. 
Note that the assertions of Lemmas $2-5$ are required only for $\ell \leq 17$ in the proof of the Theorem. We shall consider equations of the form

$$
m(m+i)(m+j)=b y^{\ell} \quad \text { in positive integers } m, y \text { with } \operatorname{gcd}(y, 5)=1
$$

where

$$
1 \leq i<j \leq 5 \quad \text { and } \quad P(b) \leq 3
$$

such that

$$
(m, i, j) \notin\{(1,1,2),(2,1,2),(2,2,4),(4,2,4)\} .
$$

We write

$$
\begin{array}{ll}
m+\mu=a_{\mu} y_{\mu}^{\ell}=A_{\mu} Y_{\mu}^{\ell} \quad & \text { with } a_{\mu} \text { lth power free, } P\left(a_{\mu}\right) \leq 3, \\
& P\left(A_{\mu}\right) \leq 3, \operatorname{gcd}\left(Y_{\mu}, 6\right)=1 \text { for } \mu \in\{0, i, j\} .
\end{array}
$$

We also write $A_{\mu}=2^{f_{\mu}} 3^{g_{\mu}}$. By (8), we observe that $\operatorname{gcd}\left(Y_{\mu}, Y_{\nu}\right)=1$ for $\mu \neq \nu, \mu, \nu \in\{0, i, j\}$. We see that the $a_{\mu}$ 's are distinct. For, otherwise we have $a_{\mu}=a_{\nu}$ for some $\mu, \nu \in\{0, i, j\}$. We may assume without loss of generality that $\mu>\nu$. Thus $y_{\mu}>y_{\nu}$ and

$$
\begin{aligned}
5 & \geq \mu-\nu=(n+\mu)-(n+\nu)=a_{\mu}\left(y_{\mu}^{\ell}-y_{\nu}^{\ell}\right) \\
& \geq a_{\mu}\left(y_{\mu}^{\ell-1}+y_{\mu}^{\ell-2} y_{\nu}+\ldots+y_{\nu}^{\ell-1}\right) \geq 6,
\end{aligned}
$$

which is a contradiction. Similarly, we see that $A_{\mu}$ 's are distinct. Further, by (8), we see that among $A_{0}, A_{i}$ and $A_{j}$, at least one is even and we conclude from Lemma 5 that at most two are even. Let $\mu_{0}, \mu_{1} \in\{0, i, j\}$ be such that $f_{\mu_{0}}>0$ is maximal and $f_{\mu_{1}}=0$. We denote the remaining element in $\{0, i, j\}$ by $\mu_{2}$. Since $j \leq 5$, we see that

$$
f_{\mu_{2}} \leq 2
$$

We apply Lemma 3 to get

Lemma 6. Let $\ell \geq 5$. Then equation (5) with (6) and (7) implies that $f_{\mu_{0}} \leq 3+f_{\mu_{2}}$.

Proof. Suppose that equation (5) with (6) and (7) is satisfied. Let $\left|\mu_{0}-\mu_{2}\right|=5$. Then $\left|\mu_{0}-\mu_{1}\right| \leq 4$ and we have

$$
2^{f_{\mu_{0}}} 3^{g_{\mu_{0}}} Y_{\mu_{0}}^{\ell}-3^{g_{\mu_{1}}} Y_{\mu_{1}}^{\ell}=\mu_{0}-\mu_{1} \text {. }
$$

Defining $g_{0,1}=\min \left(g_{\mu_{0}}, g_{\mu_{1}}\right)$, we get

$$
2^{f_{\mu_{0}}} 3^{g_{\mu_{0}}-g_{0,1}} Y_{\mu_{0}}^{\ell}-3^{g_{\mu_{1}}-g_{0,1}} Y_{\mu_{1}}^{\ell}=\frac{\mu_{0}-\mu_{1}}{3^{g_{0,1}}}
$$

with the two terms on the left hand side coprime. Since $\left|\mu_{0}-\mu_{1}\right| \leq 4$, we see that $\left(\mu_{0}-\mu_{1}\right) / 3^{g_{0,1}}$ is a power of 2 . Hence we conclude from Lemma 3 that $f_{\mu_{0}} \leq 3$. Now we suppose that $\left|\mu_{0}-\mu_{2}\right|<5$. Then

$$
2^{f_{\mu_{0}}} 3^{g_{\mu_{0}}} Y_{\mu_{0}}^{\ell}-2^{f_{\mu_{2}}} 3^{g_{\mu_{2}}} Y_{\mu_{2}}^{\ell}=\mu_{0}-\mu_{2} .
$$


If $g_{0,2}=\min \left(g_{\mu_{0}}, g_{\mu_{2}}\right)$, then

$$
2^{f_{\mu_{0}}-f_{\mu_{2}}} 3^{g_{\mu_{0}}-g_{0,2}} Y_{\mu_{0}}^{\ell}-3^{g_{\mu_{2}}-g_{0,2}} Y_{\mu_{2}}^{\ell}=\frac{\mu_{0}-\mu_{2}}{2^{f_{\mu_{2}}} 3^{g_{0,2}}}
$$

with the two terms on the left hand side coprime. Since $\left|\mu_{0}-\mu_{2}\right| \leq 4$, the right hand side is composed of 2's and 3's. Hence by Lemma $3, f_{\mu_{0}}-f_{\mu_{2}}$ $\leq 3$.

For the next assertion on equation (5), we state the following two results on Catalan equation and its extension.

LEMMA 7. The solutions of

$$
3^{m}-2^{n}= \pm 1 \quad \text { in non-negative integers } m, n
$$

are given by $(m, n) \in\{(0,1),(1,1),(2,3),(1,2)\}$.

LEMMA 8. The solutions of

$$
3^{m}-2^{n}= \pm 5 \quad \text { in non-negative integers } m, n
$$

are given by $(m, n) \in\{(1,3),(2,2),(3,5)\}$.

Lemma 7 is a well known result of Leo Hebrews and Levi Ben Gerson (see Ribenboim [8]) and Lemma 8 is due to Herschfeld [7].

LEMMA 9. Suppose that equation (5) with (6) and (7) is satisfied. Assume that either at least two distinct $Y_{\mu}, Y_{\nu}$ or two distinct $y_{\mu}, y_{\nu}$ are equal to one. Then the left hand side of equation (5) equals one of the products in the following set:

$\{1 \cdot 2 \cdot 4,1 \cdot 2 \cdot 6,1 \cdot 3 \cdot 4,1 \cdot 3 \cdot 6,1 \cdot 4 \cdot 6,2 \cdot 3 \cdot 6,3 \cdot 4 \cdot 6,3 \cdot 4 \cdot 8$,

$$
3 \cdot 6 \cdot 8,4 \cdot 6 \cdot 9,4 \cdot 8 \cdot 9,6 \cdot 8 \cdot 9,8 \cdot 9 \cdot 12\} \text {. }
$$

Proof. Suppose that equation (5) holds with (6) and (7). Let $Y_{\mu}=Y_{\nu}=1$ for some $\mu, \nu \in\{0, i, j\}$ with $\mu \neq \nu$. Then

$$
2^{f_{\mu}} 3^{g_{\mu}}-2^{f_{\nu}} 3^{g_{\nu}}=\mu-\nu \quad \text { with }|\mu-\nu| \leq 5 .
$$

There is no loss of generality in assuming $\mu-\nu>0$, thus we need to study the diophantine inequalities $1 \leq 2^{f_{\mu}} 3^{g_{\mu}}-2^{f_{\nu}} 3^{g_{\nu}} \leq 5$. We split the study according to the values of $g_{\mu}, g_{\nu}$.

1. If $g_{\mu}=g_{\nu}=0$, then $1 \leq 2^{f_{\mu}}-2^{f_{\nu}} \leq 5$ and one easily sees that $\left(f_{\mu}, f_{\nu}\right) \in\{(1,0),(2,0),(2,1),(3,2)\}$.

2. If $g_{\mu}$ and $g_{\nu}$ are non-zero, then one has $2^{f_{\mu}} 3^{g_{\mu}}-2^{f_{\nu}} 3^{g_{\nu}}=3$ and hence $2^{f_{\mu}} 3^{g_{\mu}-1}-2^{f_{\nu}} 3^{g_{\nu}-1}=1$. Then there are two possibilities:

- $f_{\mu} \neq 0$; then $f_{\nu}=0$. Then $g_{\mu}=1$, so that the solutions are (see Lemma 7) $\left(f_{\mu}, f_{\nu}, g_{\mu}, g_{\nu}\right) \in\{(1,0,1,1),(2,0,1,2)\}$.

- $f_{\nu} \neq 0$; then $f_{\mu}=0$. Similarly, the solutions are $\left(f_{\mu}, f_{\nu}, g_{\mu}, g_{\nu}\right) \in$ $\{(0,1,2,1),(0,3,3,1)\}$. 
3. If $g_{\mu} \neq 0$ and $g_{\nu}=0$, then if $f_{\nu}=0$, we have either $\left(f_{\mu}, f_{\nu}, g_{\mu}, g_{\nu}\right)=$ $(0,0,1,0)$ or $2^{f_{\mu}} 3^{g_{\mu}}-2^{f_{\nu}} \geq 5$, with equality only for $\left(f_{\mu}, f_{\nu}, g_{\mu}, g_{\nu}\right)=$ $(1,0,1,0)$. In what follows, $f_{\nu} \neq 0$. Then

- the right hand side cannot be 3 ;

- if the right hand side is 5 , then $f_{\mu}=0$ and $\left(f_{\mu}, f_{\nu}, g_{\mu}, g_{\nu}\right)=(0,2,2,0)$ from Lemma 8;

- the equation $2^{f_{\mu}} 3^{g_{\mu}}-2^{f_{\nu}} \in\{1,2,4\}$ reduces to the case $2^{f_{\mu}} 3^{g_{\mu}}-$ $2^{f_{\nu}}=4$. Then $f_{\mu}=f_{\nu}$ or $\min \left(f_{\mu}, f_{\nu}\right)=2$. The former case yields $\left(f_{\mu}, f_{\nu}, g_{\mu}, g_{\nu}\right)=(1,1,1,0)$, whereas the latter yields $f_{\mu}=2$, and then $3^{g_{\mu}}-2^{f_{\nu}-2}=1$, thus $\left(f_{\mu}, f_{\nu}, g_{\mu}, g_{\nu}\right) \in\{(2,3,1,0),(2,5,2,0)\}$. The solutions for $2^{f_{\mu}} 3^{g_{\mu}}-2^{f_{\nu}}=1,2$ are $\{(0,1,1,0),(0,3,2,0)\}$ and $\{(1,2,1,0),(1,4,2,0)\}$, respectively.

4. If $g_{\nu} \neq 0$ and $g_{\mu}=0$, it is easily seen that $f_{\mu} \neq 0$. The study is then identical to the preceding case, except that we use the "minus" cases of Lemmas 7 and 8 . We get $\left(f_{\mu}, f_{\nu}, g_{\mu}, g_{\nu}\right) \in\{(3,0,0,1),(5,0,0,3),(4,2,0,1)$, $(2,0,0,1),(3,1,0,1)\}$.

To sum up, we get $(m+\mu, m+\nu) \in\{(2,1),(4,1),(4,2),(8,4),(6,3)$, $(12,9),(9,6),(27,24),(3,1),(6,1),(9,4),(6,2),(12,8),(36,32),(3,2),(9,8)$, $(6,4),(18,16),(8,3),(32,27),(16,12),(4,3),(8,6)\}$.

For each of these pairs $(\alpha, \beta)$ with $\alpha>\beta$, the corresponding possible products in (5) are obtained by taking (up to permutation) the triples $(\alpha, \beta, \gamma)$ with $\gamma>0$ and $\alpha-5 \leq \gamma \leq \beta+5$ and $P(\gamma) \leq 3$, and excluding (cf. (7)) $(1,2,3),(2,3,4),(2,4,6),(4,6,8)$, we obtain the assertion of the lemma. Since $Y_{\mu}$ divides $y_{\mu}$ and $Y_{\nu}$ divides $y_{\nu}$, as is seen from the definition, the result follows in the case $y_{\mu}=y_{\nu}=1$.

Lemma 10. Let $\ell \geq 5$. Assume that equation (5) with (6) and (7) is satisfied. Suppose that there is at most one $Y_{\mu}$ which equals one. Then there exists $\left(Z_{1}, Z_{2}\right) \in\left\{Y_{\mu_{0}}, Y_{\mu_{1}}, Y_{\mu_{2}}\right\}$ such that

$$
a Z_{1}^{\ell}-b Z_{2}^{\ell}= \pm c
$$

for some $a, b, c$ given below:

$$
\begin{array}{ll}
b=1, & a \in\{2,4,8\}, c \in\{3,5\} ; \\
b=1, & a=3, c \in\{1,2,4,5\} ; \\
b=1, & a \in\{6,12,24\}, c \in\{1,5\} ; \\
b=1, & a \in\{48,96\}, c=5 ; \\
b=3, & a \in\{2,4,8\}, c \in\{1,5\} ; \\
b=3, & a \in\{16,32\}, c=5 .
\end{array}
$$


Proof. For any $\mu \neq \nu \in\{0, i, j\}$, we have $2^{f_{\mu}} 3^{g_{\mu}} Y_{\mu}^{\ell}-2^{f_{\nu}} 3^{g_{\nu}} Y_{\nu}^{\ell}=\mu-\nu$. We shall prove, using Lemmas 2 and 3, that only a finite number of such diophantine equations need to be considered, namely, those just above.

First, since $|\mu-\nu| \leq 5$, we see that at most two of $g_{0}, g_{i}, g_{j}$ can be non-zero and that at most one can be larger than 2 .

Assume that there are $\mu, \nu$ such that $g_{\mu}=g_{\nu}=0$. Then we have $2^{f_{\mu}} Y_{\mu}^{\ell}-$ $2^{f_{\nu}} Y_{\nu}^{\ell}=\mu-\nu$. Dividing by a suitable power of 2 , we can assume without loss of generality that $f_{\nu}=0$ and hence $\mu-\nu$ is odd; Lemma 2 then shows (since $\left.\left(Y_{\mu}, Y_{\nu}\right) \neq(1,1)\right)$ that $\mu-\nu \in\{ \pm 3, \pm 5\}$. Using Lemma 3 , we see that $f_{\mu} \leq 3$, which gives $b=1, a \in\{2,4,8\}, c \in\{3,5\}$.

Assume now that $g_{\mu}$ and $g_{\mu^{\prime}}$ are non-zero. If $\{0, i, j\}=\left\{\mu, \mu^{\prime}, \nu\right\}$, then $g_{\nu}=0$. Furthermore, one of $g_{\mu}$ and $g_{\mu^{\prime}}$, say $g_{\mu}$, is equal to 1 . Thus we have $2^{f_{\mu}} 3 Y_{\mu}^{\ell}-2^{f_{\nu}} Y_{\nu}^{\ell}=\mu-\nu$ and dividing by a suitable power of 2 , we can assume that $\min \left(f_{\mu}, f_{\nu}\right)=0$. The fact that $\left(Y_{\nu}, 6\right)=1$ shows that $\mu-\nu \neq \pm 3$. If $f_{\mu}=f_{\nu}=0$, we get $a=3, b=1, c \in\{1,2,4,5\}$. Otherwise $\mu-\nu$ is odd; then Lemma 3 shows that $\max \left(f_{\mu}, f_{\nu}\right) \leq 3$ if $\mu-\nu= \pm 1$, whereas Lemma 6 shows that $\max \left(f_{\mu}, f_{\nu}\right) \leq 5$ if $\mu-\nu \neq \pm 1$. This yields all the remaining cases.

We prove an assertion similar to Lemma 10 for the case $\ell=3$ using the following result of Nagell and Ljunggren (see Ribenboim [8, pp. 96-105]).

LEMma 11. The only non-zero solutions of the equations

$$
x^{2}+x+1=y^{3} \quad \text { and } \quad x^{2}+x+1=3 y^{3}
$$

in integers $x, y$ are given by

$$
(x, y)=(-1,1),(18,7),(-19,7)
$$

and

$$
(x, y)=(1,1)
$$

respectively.

Lemma 12. Let $\ell=3$. Assume that equation (5) with (6) and (7) is satisfied. Suppose there is at most one $y_{\mu}$ which equals one. Then there exists $\left(Z_{1}, Z_{2}\right)=\left(y_{\mu}, y_{\nu}\right)$ with $y_{\mu}, y_{\nu} \in\left\{y_{\mu_{0}}, y_{\mu_{1}}, y_{\mu_{2}}\right\}$ such that

$$
a Z_{1}^{3}-b Z_{2}^{3}= \pm c
$$

for some $a, b, c$ given below:

$$
\begin{array}{ll}
b=1, & a \in\{2,4,6,12\}, c \in\{3,5\} ; \\
b=1, & a \in\{3,9\}, c \in\{2,4,5\} ; \\
b=1, & a \in\{18,36\}, c=5 ; \\
b=3, & a \in\{2,4\}, c=5 ; \\
b=9, & a \in\{2,4\}, c \in\{1,5\} .
\end{array}
$$

Further $(m+\mu, m+\nu)=\left(a Z_{1}^{3}, b Z_{2}^{3}\right)$. 
Proof. We suppose that equation (5) with (6) and (7) is satisfied. Since $a_{\mu_{1}}$ is odd, we have $a_{\mu_{1}} \in\left\{1,3,3^{2}\right\}$. Further $a_{\mu_{2}} \in\{1,2,3,4,6,9,12,18,36\}$.

- Let $a_{\mu_{1}}=1$. We consider $a_{\mu_{2}} y_{\mu_{2}}^{3}-y_{\mu_{1}}^{3}=\mu_{2}-\mu_{1}$.

Let $a_{\mu_{2}} \in\{2,4,6,12\}$. Then $\mu_{2}-\mu_{1} \in\{ \pm 1, \pm 3, \pm 5\}$ since $y_{\mu_{1}}$ is odd. Suppose $\mu_{2}-\mu_{1}=1$. Then $y_{\mu_{1}}^{3}+1=a_{\mu_{2}} y_{\mu_{2}}^{3}$. Hence $\left(y_{\mu_{1}}^{3}+1\right) /\left(y_{\mu_{1}}+1\right)$ is either $y_{\mu_{2}}^{\prime 3}$ or $3 y_{\mu_{2}}^{\prime 3}$. Now we apply Lemma 11 to see that $y_{\mu_{1}}$ is either 1 or 19. If $m+\mu_{1}=1$, then $m+\mu_{2}=2$. Thus $y_{\mu_{1}}=y_{\mu_{2}}=1$, which is excluded. If $m+\mu_{1}=19^{3}$, we check that $m+\mu_{1}+1$ is not of the form $a_{\mu_{2}} y_{\mu_{2}}^{3}$ with $a_{\mu_{2}} \in\{2,4,6,12\}$. Thus there is no possible value for $m+\mu_{2}$. Hence $\mu_{2}-\mu_{1} \neq 1$. The possibility $\mu_{2}-\mu_{1}=-1$ is excluded similarly. Thus we see that $y_{\mu_{2}}, y_{\mu_{1}}$ is a solution of (10) with $b=1, a \in\{2,4,6,12\}, c \in\{3,5\}$.

Let $a_{\mu_{2}} \in\{3,9\}$. Then we apply Lemma 11 to see that $\mu_{2}-\mu_{1} \neq \pm 1, \pm 3$. Thus $\left(y_{\mu_{2}}, y_{\mu_{1}}\right)$ is a solution of (10) with $b=1, a \in\{3,9\}, c \in\{2,4,5\}$.

Let $a_{\mu_{2}} \in\{18,36\}$. Since $y_{\mu_{1}}$ is odd, $\mu_{2}-\mu_{1} \in\{ \pm 1, \pm 3, \pm 5\}$. Now $\mu_{2}-\mu_{1} \neq \pm 3$ since otherwise $3 \mid y_{\mu_{1}}$ implying $9 \mid\left(\mu_{2}-\mu_{1}\right)$, a contradiction. Further by Lemma $11, \mu_{2}-\mu_{1} \neq \pm 1$. Thus $\left(y_{\mu_{2}}, y_{\mu_{1}}\right)$ is a solution of (10) with $b=1, a \in\{18,36\}$ and $c=5$.

- Let $a_{\mu_{1}}=3$. Suppose $a_{\mu_{2}}=1$. We use Lemma 11 to see that $\mu_{1}-\mu_{2}=$ $\pm 2, \pm 4, \pm 5$. Thus $\left(y_{\mu_{1}}, y_{\mu_{2}}\right)$ is a solution of (10) with $b=1, a=3, c \in$ $\{2,4,5\}$.

Suppose $a_{\mu_{2}}=2$ or 4 . Then we use Lemmas 4 and 11 to see that $\mu_{2}-\mu_{1}=$ \pm 5 . Thus $\left(y_{\mu_{2}}, y_{\mu_{1}}\right)$ is a solution of (10) with $b=3, a \in\{2,4\}, c=5$.

Similarly, we apply Lemma 11 to exclude $a_{\mu_{2}} \in\{6,9,12,18,36\}$.

- Let $a_{\mu_{1}}=9$. We argue as in the case $a_{\mu_{1}}=3$ to see that $\left(y_{\mu_{1}}, y_{\mu_{2}}\right)$ is a solution of (10) with $b=1, a=9, c \in\{2,4,5\}$ or $\left(y_{\mu_{2}}, y_{\mu_{1}}\right)$ is a solution of (10) with $b=9, a \in\{2,4\}, c=\{1,5\}$.

Finally the relation $(m+\mu, m+\nu)=\left(a Z_{1}^{3}, b Z_{2}^{3}\right)$ is clear from the proof.

For a Thue equation of the form $A x^{\ell}-B y^{\ell}= \pm C$ in non-negative integers $x, y$, we say that a solution $(x, y)$ is a trivial solution if $\max (x, y) \leq 1$.

Lemma 13. Let $5 \leq \ell \leq 17$. The set of equations in (9) has no non-trivial solution.

LEMma 14. Let $\ell=3$. The only non-trivial solutions of the set of equations in (10) are given by

$$
\begin{array}{r}
\left(a, b, c ; Z_{1}, Z_{2}\right) \in\{(1,2,-3 ; 5,4),(1,3,5 ; 2,1),(1,4,-5 ; 3,2) \\
(1,6,5 ; 467,257),(3,2,5 ; 7,8)\} .
\end{array}
$$

Proofs of Lemmas 13 and 14. The proof of Lemmas 13 and 14 rests on a mix of modular arguments (for most of the equations that do not have solutions at all) and effective solution of Thue equations, as e.g. in [2]. We give a short overview of these techniques now. 
In the proof, $x^{1 / \ell}$ and $\log x$ denote the principal determination of the corresponding functions.

Rewrite the Thue equation $a X^{\ell}-b Y^{\ell}=c$ as $X^{\ell}-\alpha Y^{\ell}=\beta$, where $\alpha$ is a positive integer, not an $\ell$ th power. This can be rewritten as

$$
\prod_{\zeta^{\ell}=1}\left(X-\zeta \alpha^{1 / \ell} Y\right)=\beta
$$

Let $\mathbb{K}$ be the number field $\mathbb{Q}\left(\alpha^{1 / \ell}\right)$, and let $M$ be a complete set of nonassociate solutions of the norm equation $N_{\mathbb{K} / \mathbb{Q}}(\mu)=\beta$. Then for any solution $(X, Y)$, there is a $\mu$ in $M$ and a unit $\eta$ of $\mathbb{K}$ such that $X-\alpha^{1 / \ell} Y=\mu \eta$.

We now split the overview in two cases, according to the value of $\ell$.

First, when $\ell \leq 7$, a system of fundamental units may be easily computed using the PARI library. Thus, we can compute $\eta_{1}, \ldots, \eta_{r}$ and there exist integers $b_{1}, \ldots, b_{r}$ such that

$$
X-\alpha^{1 / \ell} Y=\mu \eta_{1}^{b_{1}} \ldots \eta_{r}^{b_{r}}
$$

Taking logarithms of all the conjugates of this equation shows that

$$
\log \left(X-\sigma_{k}\left(\alpha^{1 / \ell}\right) Y\right)=\log \sigma_{k}(\mu)+\sum_{i=1}^{r} b_{i} \log \sigma_{k}\left(\eta_{i}\right)+b_{0, k} i \pi
$$

with $b_{0, k}=O\left(\max _{i}\left|b_{i}\right|\right)$.

Elementary arguments allow one to prove that for any $\sigma_{k}, \sigma_{k^{\prime}} \neq \mathrm{id}$, one has

$$
\left|\log \frac{X / Y-\sigma_{k}\left(\alpha^{1 / \ell}\right)}{\alpha^{1 / \ell}-\sigma_{k}\left(\alpha^{1 / \ell}\right)}\right| \ll \exp \left(-C \max _{i}\left|b_{i}\right|\right) \asymp|Y|^{-\ell}
$$

and in particular

$$
\begin{array}{r}
\left|\log \left(\frac{\alpha^{1 / \ell}-\sigma_{k^{\prime}}\left(\alpha^{1 / \ell}\right)}{\alpha^{1 / \ell}-\sigma_{k}\left(\alpha^{1 / \ell}\right)} \cdot \frac{X-\sigma_{k}\left(\alpha^{1 / \ell}\right) Y}{X-\sigma_{k^{\prime}}\left(\alpha^{1 / \ell}\right) Y}\right)\right| \\
\ll \exp \left(-C \max _{i}\left|b_{i}\right|\right) \asymp|Y|^{-\ell}
\end{array}
$$

where both $C$ and the implicit constants are effective.

Now, (11) shows that the left hand side of (13) can be rewritten as a linear form in logarithms of algebraic numbers. As such, it can be bounded from below by Baker's method (note that if the linear form is zero, then $\alpha^{1 / \ell}=X / Y$ is a rational), with a bound $\exp \left(-C^{\prime} \log \max _{i}\left|b_{i}\right|\right)$. Comparing this with the upper bound provides us with a (very large) upper bound on $\max _{i}\left|b_{i}\right|$.

We need to improve this bound. Since the upper bound in (13) does not leave much place for improvement, we need to replace Baker's bound by a better one, using the restriction on the $b_{i}$, i.e., to bound from below a linear form $\sum_{i=1}^{r} b_{i} \theta_{i}+\psi$, under the assumption that $\left|b_{i}\right| \leq B$ for a very 
large $B$. We can in what follows forget about the arithmetical nature of the coefficients $\theta_{i}$, only their numerical properties will be relevant.

This improvement of the bound can be achieved by means of effective diophantine approximation techniques, such as computing continued fractions (when $r=2$ ) or the LLL algorithm. We first show how one can reduce to the case $r=2$ by applying Bilu and Hanrot's method [2].

First note that when $\ell=3$, taking the imaginary part of (13) yields a linear form with $r=2$. Otherwise, let us order the embeddings so that $\sigma_{0}=\mathrm{id}, \sigma_{r+i}=\bar{\sigma}_{i}$. Take the real part of the identity (11):

$$
\log \left|\left(X-\sigma_{k}\left(\alpha^{1 / \ell}\right) Y\right)\right|=\log \left|\sigma_{k}(\mu)\right|+\sum_{i=1}^{r} b_{i} \log \left|\sigma_{k}\left(\eta_{i}\right)\right|
$$

which becomes, if we apply (12),

$$
\log |Y|=\log \left|\sigma_{k}(\mu)\right|-\log \left|\alpha^{1 / \ell}-\sigma_{k}\left(\alpha^{1 / \ell}\right)\right|+\sum_{i=1}^{r} b_{i} \log \left|\sigma_{k}\left(\eta_{i}\right)\right|+O\left(|Y|^{-\ell}\right) .
$$

Let $A=\left[a_{i j}\right]_{1 \leq i, j \leq r-1}$ be the inverse of the matrix $\left[\sigma_{j}\left(\eta_{i}\right)\right]_{1 \leq i, j \leq r-1}$. The determinant of this matrix is, up to a power of 2 , equal to the regulator of the field, so that this matrix is indeed invertible.

Then one has

$$
b_{i}=\log |Y| \sum_{j=1}^{r} a_{i j}+\sum_{j=1}^{r} a_{i j} \log \left|\frac{\alpha^{1 / \ell}-\sigma_{j}\left(\alpha^{1 / \ell}\right)}{\sigma_{j}(\mu)}\right|+O\left(|Y|^{-\ell}\right) .
$$

Put $\delta_{i}=\sum_{j=1}^{r} a_{i j}$ and $\lambda_{i}=\sum_{j=1}^{r} a_{i j} \log \left|\left(\alpha^{1 / \ell}-\sigma_{j}\left(\alpha^{1 / \ell}\right)\right) / \sigma_{j}(\mu)\right|$. Then we can eliminate $\log |Y|$ by combining identities (14) for two different values of $i$. More precisely, define $\delta=\delta_{i_{1}} \delta_{i_{2}}^{-1}$ and $\lambda=\delta \lambda_{i_{2}}-\lambda_{i_{1}}$. Then we get the estimate $\left|b_{i_{2}}+b_{i_{1}} \delta+\lambda\right| \ll \exp \left(-C \max _{i}\left|b_{i}\right|\right) \asymp|Y|^{-\ell}$.

Thus, we have to estimate an expression of the kind $\left|b_{1}+b_{2} \delta+\lambda\right|$ from below. We denote by $\|x\|$ the distance from $x$ to $\mathbb{Z}$. Let $q$ be the denominator of a convergent of the continued fraction expansion of $\delta$; choose $q$ slightly larger than the bound $B$ on $\max _{i}\left|b_{i}\right|$, say $q=\kappa B$. Then one has

$$
q\left|b_{1}+b_{2} \delta+\lambda\right| \geq\left\|q b_{2} \delta+q \lambda\right\| \geq\left|\|q \lambda\|-b_{2}\|q \delta\|\right| \geq\left|\|q \lambda\|-\kappa^{-1}\right| .
$$

If $\|q \lambda\|$ is not too small, we see that this gives us a lower bound for $\left|b_{1}+b_{2} \delta+\lambda\right|$. This lower bound in turn can be used to obtain a new bound on the $b_{i}$ and so on. When we reach a sufficiently small bound (usually in two steps), we enumerate all the possible $r$-tuples $\left(b_{1}, \ldots, b_{r}\right)$. Recall that in that case $r \leq 3$.

Second, if $\ell \geq 11$, PARI can still give a system of units, but ensuring that this system is fundamental without assuming the GRH may take a lot of time. Using lower bounds for regulators (e.g. [15]) we can however compute 
an upper bound $\mathcal{B}$ of the index of our system of units $\eta_{1}, \ldots, \eta_{r}$. Hence there exists $b_{0} \leq \mathcal{B}$ and $b_{1}, \ldots, b_{r}$ such that

$$
\left(X-\alpha^{1 / \ell} Y\right)^{b_{0}}=\mu^{b_{0}} \eta_{1}^{b_{1}} \ldots \eta_{r}^{b_{r}} .
$$

All the treatment is then very similar to the previous case except that one can no more reduce the "effective estimation problem" to continued fractions; one has to use the 3-dimensional version of the LLL algorithm instead. See [6] for more details.

In that case, it is difficult to reduce the bound on the $b_{i}$ to a reasonable value and the high value of the rank $r=(\ell-1) / 2$ may make a complete enumeration of all the $(r+1)$-tuples $\left(b_{0}, b_{1}, \ldots, b_{r}\right)$ tricky. It is far better to use the lower bound on the linear form that we just derived in association with the rightmost part of (13). This yields directly a bound on $|Y|$ which is very small when $\ell$ is large. Enumerating the corresponding values is then just a matter of routine.

3. Proof of the Theorem. Suppose that equation (1) with $r=1$ and $n>k^{\ell}$ is satisfied. Let $6 \leq k \leq 8$. For $1 \leq i \leq t$, we write

$$
n+d_{i}=a_{i} x_{i}^{\ell}, \quad a_{i} \text { is } \ell \text { th power free, } P\left(a_{i}\right) \leq k .
$$

Since $n>k^{\ell}$ we see that $a_{i}$ 's are distinct. Further Lemma 1 holds. We observe that $f_{0}(k, 2) \geq 3$ and $f_{0}(k, 3) \geq 5$. We apply Lemma 1 with $\ell^{\prime}=\ell-1$ and $m=3$ to obtain $\left(\begin{array}{c}\ell+3 \\ \ell-1\end{array}\right) \leq \ell^{3}$. This inequality is not satisfied when $\ell=19$. By induction, it is also not satisfied for every odd $\ell \geq 19$. Hence we conclude from Lemma 1 that $\ell \leq 17$. If $f_{0}(k, 2) \geq 4$, we apply Lemma 1 with $\ell^{\prime}=\ell-1$ and $m=2$ to derive that $\left(\begin{array}{c}\ell+2 \\ \ell-1\end{array}\right) \leq \ell^{2}$, which is not valid for $\ell \geq 3$. Hence $f_{0}(k, 2)=3$.

Let $k=8$. Since $f_{0}(k, 2)=3$, we see that 7 divides $a_{0}, a_{7} ; 5$ divides $a_{1}, a_{6}$ and by Lemma 5 , the omitted term is either $n+3$ or $n+4$. Thus we have two possible equations:

$$
n(n+1)(n+2)(n+4)(n+5)(n+6)(n+7)=b y^{\ell}
$$

with

$$
(n+2)(n+4)(n+5)=b^{\prime} y^{\prime \ell}, \quad P\left(b^{\prime}\right) \leq 3,
$$

or

$$
n(n+1)(n+2)(n+3)(n+5)(n+6)(n+7)=b y^{\ell}
$$

with

$$
(n+2)(n+3)(n+5)=b^{\prime} y^{\prime \ell}, \quad P\left(b^{\prime}\right) \leq 3 .
$$

We find that (16) and (18) are equations of the form (5) with $m=n+2$, $i=2, j=3$ and $m=n+2, i=1, j=3$, respectively. Thus (6) and (7) are satisfied. We use the fact that $7 \mid n$ and $5 \mid(n+1)$ to deduce from Lemma 9 that at most one $Y_{\mu}$ equals 1 . Hence by Lemmas 10 and 13, we conclude that 
equations (16) and (18) have no solution for $\ell \geq 5$. Let $\ell=3$. By Lemma 9 , we have at most one $y_{\mu}$ equalling 1 . Then by Lemmas 10 and 14 , we see that $(n+2, n+5)=(125,128)$. This is not possible since $5 \mid(n+1)$. Thus equations (16) and (18) do not hold. This excludes the case $k=8$.

Let $k=6$. Then we have 5 dividing $a_{0}, a_{5}$ and the omitted term is either $n+2$ or $n+3$. Thus we have either

$$
n(n+1)(n+3)(n+4)(n+5)=b y^{\ell}
$$

with

$$
(n+1)(n+3)(n+4)=b^{\prime} y^{\prime \ell}
$$

or

$$
n(n+1)(n+2)(n+4)(n+5)=b y^{\ell}
$$

$$
(n+1)(n+2)(n+4)=b^{\prime} y^{\prime \ell}, \quad P\left(b^{\prime}\right) \leq 3 .
$$

We proceed as in the case $k=8$ and conclude that equations (20) and (22) are impossible.

Let $k=7$. Then 5 divides $a_{0}$ and $a_{5}$ or $a_{1}$ and $a_{6}$. By excluding the omitted term and the term divisible by 7 , we arrive at equations of the form (5) with (6) and (7). We discuss one case. The arguments for other cases are similar. We take the case when 5 divides $a_{1}$ and $a_{6}, 7$ divides $a_{2}$ and the omitted term is $n+4$. Then we get

$$
n(n+3)(n+5)=b^{\prime} y^{\prime \ell}, \quad P\left(b^{\prime}\right) \leq 3, \quad \operatorname{gcd}(y, 5)=1 .
$$

By Lemma 9 we find that if at least two distinct $Y_{\mu}, Y_{\nu}$ or $y_{\mu}, y_{\nu}$ are equal to 1 , then $n=1$ or 3 , which contradicts $5 \mid(n+1)$. Thus we may assume that at most one $Y_{\mu}$ or $y_{\nu}$ equals 1 . Then by Lemmas 10,12,13, 14, we get $\ell=3$ and

$$
(n, n+3)=(125,128)
$$

or

$$
(n, n+5) \in\left\{(3,8),(27,32),\left(467^{3}, 6 \cdot 257^{3}\right),\left(3 \cdot 7^{3}, 2 \cdot 8^{3}\right) .\right.
$$

These possibilities are ruled out since $5 \mid(n+1)$ and $7 \mid(n+2)$.

Let $k=5, P(b)<k$. Then $f_{0}(k, 2)=4$ and hence Lemma 1 does not hold with $\ell^{\prime}=\ell-1, m=2$ for $\ell \geq 3$.

Let $k=3, P(b)<k$. Then the assertion follows from Lemma 2 .

\section{References}

[1] M. Bennett, Rational approximation to algebraic numbers of small height: The diophantine equation $\left|a x^{n}-b y^{n}\right|=1$, J. Reine Angew. Math., to appear.

[2] Yu. Bilu and G. Hanrot, Solving Thue equations of high degree, J. Number Theory 60 (1996), 373-392. 
[3] H. Darmon and L. Merel, Winding quotients and some variants of Fermat's Last Theorem, J. Reine Angew. Math. 490 (1997), 81-100.

[4] P. Erdős and J. L. Selfridge, The product of consecutive integers is never a power, Illinois J. Math. 19 (1975), 292-301.

[5] K. Győry, On the diophantine equation $n(n+1) \ldots(n+k+1)=b x^{l}$, Acta Arith. 83 (1998), 87-92.

[6] G. Hanrot, Solving Thue equations without the full unit group, Math. Comp. 69 (2000), 395-405.

[7] A. Herschfeld, The equation $2^{x}-3^{y}=d$, Bull. Amer. Math. Soc. 42 (1936), 231-234.

[8] P. Ribenboim, Catalan's Conjecture, Academic Press, 1994.

[9] K. A. Ribet, On the equation $a^{p}+2^{\alpha} b^{p}+c^{p}=0$, Acta Arith. 79 (1997), 7-16.

[10] J. W. Sander, Rational points on a class of superelliptic curves, J. London Math. Soc. 59 (1999), 422-434.

[11] N. Saradha, On perfect powers in products with terms from arithmetic progressions, Acta Arith. 82 (1997), 147-172.

[12] N. Saradha and T. N. Shorey, Almost perfect powers in arithmetic progression, ibid., to appear.

[13] T. N. Shorey, Exponential diophantine equations involving products of consecutive integers and related equations, in: Number Theory, R. P. Bambah et al. (eds.), Hindustan Book Agency, 1999, 463-495.

[14] T. N. Shorey and R. Tijdeman, Some methods of Erdös applied to finite arithmetic progressions, in: The Mathematics of Paul Erdős I, R. L. Graham and J. Nešetrril (eds.), Springer, 1997, 251-267.

[15] R. Zimmert, Ideale kleiner Norm in Idealklassen und eine Regulatorabschätzung, Invent. Math. 62 (1981), 367-380.

Projet PolKA, INRIA Lorraine

615, rue du Jardin Botanique

F-54602 Villers-lès-Nancy Cedex

France

E-mail: Guillaume.Hanrot@loria.fr
School of Mathematics Tata Institute of Fundamental Research Homi Bhabha Road Mumbai 400005 India E-mail: saradha@math.tifr.res.in shorey@math.tifr.res.in

Received on 21.12.1999

and in revised form on 17.11.2000 